\title{
Case Report Meningioma of Foramen Magnum Causing Drop Attacks
}

\author{
Amit Mahore, ${ }^{1}$ Raghvendra Ramdasi, ${ }^{2}$ Sandip Mavani, ${ }^{2}$ Vithal Rangarajan, \\ Manoj Patil, ${ }^{2}$ Prashant Sathe, ${ }^{2}$ Juhi Kawale, ${ }^{3}$ and Vishakha Tikeykar ${ }^{4}$ \\ ${ }^{1}$ Department of Neurosurgery, Surana Hospital \& Research Centre, Malad, Mumbai 400064, India \\ ${ }^{2}$ Department of Neurosurgery, King Edward Memorial Hospital and Seth Gordhandas Sunderdas Medical College, \\ Parel, Mumbai 400012, India \\ ${ }^{3}$ Department of Medicine, King Edward Memorial Hospital and Seth Gordhandas Sunderdas Medical College, \\ Parel, Mumbai 400012, India \\ ${ }^{4}$ Department of Pathology, Bombay Hospital Institute of Medical Sciences \& Research, New Marine Lines, Mumbai 400020, India
}

Correspondence should be addressed to Raghvendra Ramdasi; drraghavr@gmail.com

Received 29 November 2014; Revised 20 January 2015; Accepted 6 February 2015

Academic Editor: Peter Berlit

Copyright (C) 2015 Amit Mahore et al. This is an open access article distributed under the Creative Commons Attribution License, which permits unrestricted use, distribution, and reproduction in any medium, provided the original work is properly cited.

A 52-year-old female presented with frequent episodes of falls without loss of consciousness. These episodes lasted for brief period followed by full neurological recovery. Magnetic resonance imaging (MRI) of the brain showed foramen magnum meningioma encasing left vertebral artery. The patient had dramatic improvement after excision of the tumor.

\section{Introduction}

Meningioma is the commonest tumor in the region of foramen magnum [1]. It often presents with occipitocervical pain, long tract signs, and lower cranial nerve deficits $[2,3]$. Drop attack as a presenting symptom of meningioma of foramen magnum has never been reported. We report an adult patient with this unique presentation and discuss the differential diagnosis, diagnostic approach, and a brief review of literature.

\section{Case Report}

A 52-year-old female, a known case of hypertension, presented with sudden episodes of fall without loss of consciousness for the last 5 months. Each episode used to last for approximately 1 minute followed by full neurological recovery. The frequency of such drop attacks was 1-2 per day. It was associated with giddiness. Neurological examination was normal apart from mild hyperreflexia in all four limbs.

Twenty-four-hour electroencephalography (EEG) was done by a neurologist which was normal. Electromyography (EMG) and nerve conduction velocity (NCV) of all four limbs were also normal. Cardiovascular workup which included echocardiograms (ECG) and Holter monitoring were normal. Magnetic resonance imaging (MRI) revealed anterolaterally placed, homogenously enhancing dural based lesion in the foramen magnum encasing the left vertebral artery suggestive of meningioma (Figures 1(a), 1(b), and 1(c)).

Patient underwent surgery by posterior approach. Total excision of the tumor followed by augmentation duraplasty was performed via midline suboccipital craniectomy and removal of $\mathrm{Cl}$ arch. Tumor was arising from anterolateral dura of foramen magnum, firm in consistency, vascular, and nonsuckable. It was excised in piecemeal, baring the left vertebral artery and left posterior inferior cerebellar arteries (PICA). Complete excision was done with coagulation of dural base.

Patient had a dramatic recovery from her symptoms postoperatively. At follow-up of 18 months after the surgery patient is disease-free (Figures $1(\mathrm{~d})$ and $1(\mathrm{e})$ ).

Histopathological examination showed the lesion to be meningothelial meningioma (Figure 2).

\section{Discussion}

Fall of sudden onset can be associated with or without loss of consciousness. Falls with loss of consciousness can be 


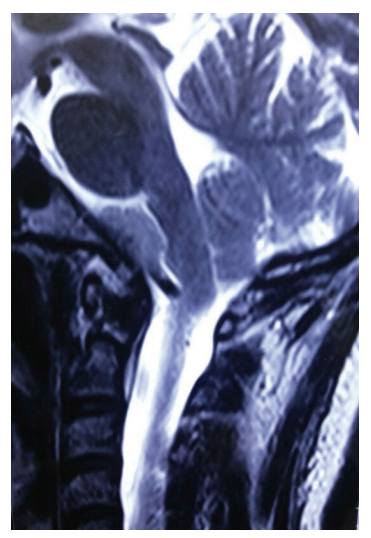

(a)

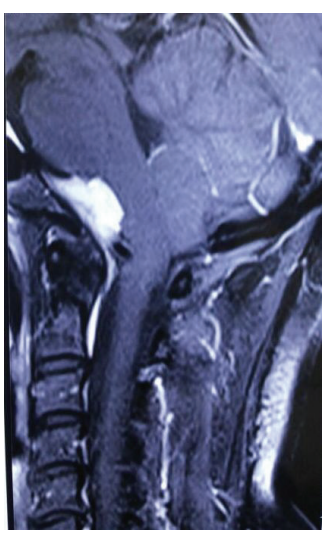

(b)

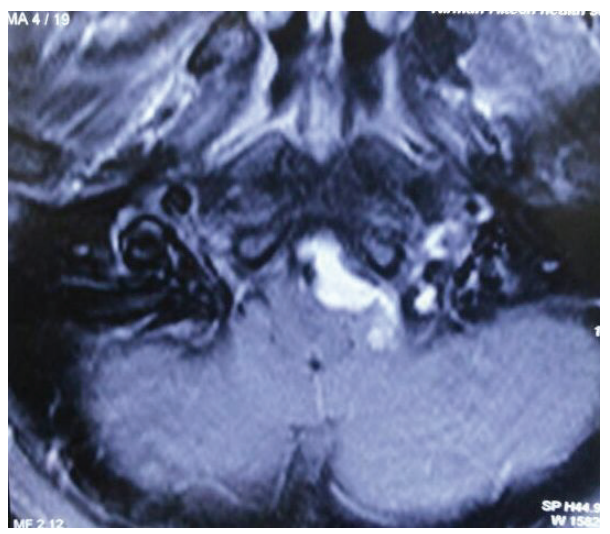

(c)

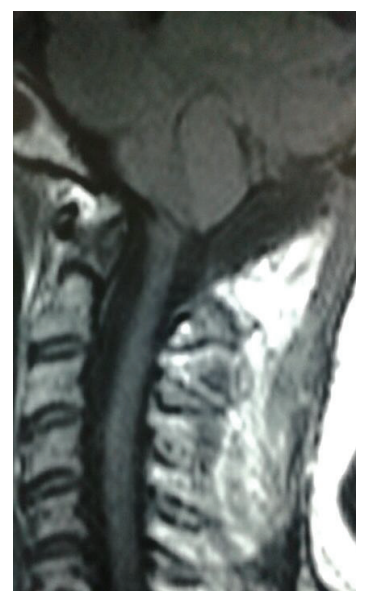

(d)

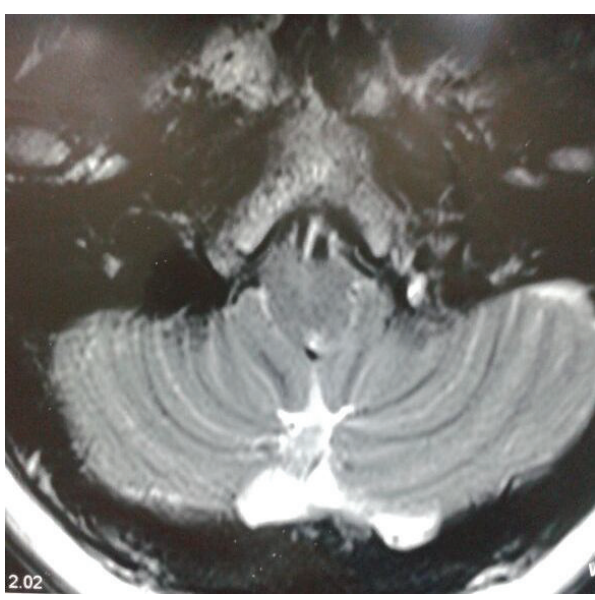

(e)

Figure 1: (a) T2 weighted sagittal image; (b) postcontrast T1 weighted sagittal image; (c) postcontrast T1 weighted axial image: magnetic resonance imaging (MRI) showing anterolaterally placed, homogenously enhancing dural based lesion in the foramen magnum encasing the left vertebral artery suggestive of meningioma. (d) T1 weighted sagittal image and (e) T1 weighted axial image: postoperative MRI showing complete excision of tumor.

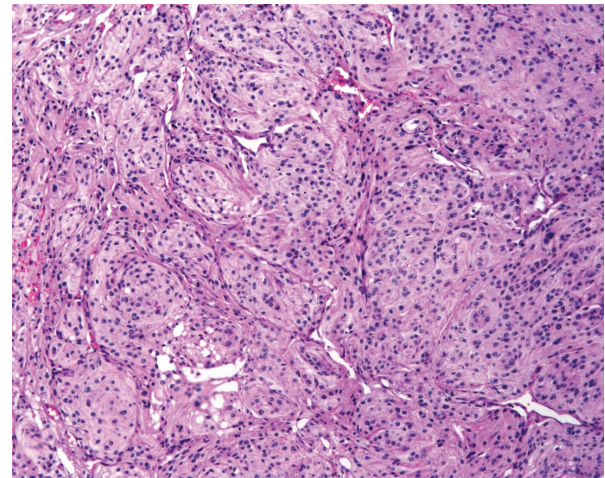

Figure 2: Photomicrograph (H\& E, 10x) showing lobular arrangement of meningothelial cells with syncytial distribution at the periphery suggestive of meningothelial meningioma.

due to syncope or nonsyncopal conditions like metabolic disorders including hypoglycaemia: hypoxia, epilepsy, and intoxications. Falls without loss of consciousness can be due

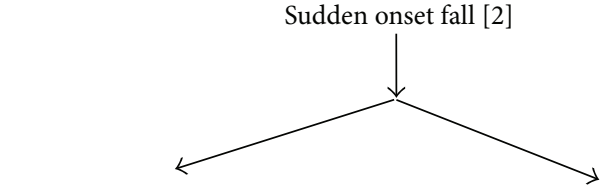

With loss of consciousness Syncope

Metabolic conditions Epilepsy Intoxications Without loss of consciousness Cataplexy Drop attack Psychogenic syncope TIA

FIGURE 3: Causes of sudden onset fall.

to cataplexy, drop attacks, psychogenic "syncope" (somatization disorders), and transient ischaemic attacks (Figure 3).

Syncope is defined as a transient, self-limited loss of consciousness, usually leading to fall. The onset of syncope is relatively rapid, and the subsequent recovery is spontaneous, complete, and usually prompt. The underlying mechanism is a transient global cerebral hypoperfusion. The causes 
TABLE 1: Tumors causing drop attack.

\begin{tabular}{lcccc}
\hline Author/year & Number & Age/sex & Site and nature of the tumor & Mechanism \\
\hline Criscuolo and Symon (1986) [4] & 1 & - & 3rd ventricular meningioma & Hydrocephalus \\
Lee et al. (1994) [5] & 1 & - & Posterior fossa arachnoid cyst & - \\
George and Laurian (1989) [6] & 1 & $31 / \mathrm{M}$ & C2 neurinoma & Vertebral \\
Pollack et al. (1995) [7] & 1 & 2/Mch & Choroid plexus papilloma third ventricle & Hydrocephalus \\
& & & Foramen magnum meningioma & Vertebral \\
Present case & 1 & $46 / \mathrm{F}$ & & insufficiency and \\
& & & & medullary \\
\end{tabular}

M: male, F: female, and Mch: male child.

of syncope include neurally mediated reflex syncopal syndromes, cardiac arrhythmias, orthostatic hypotension, and cerebrovascular conditions [8].

Drop attacks are sudden falls without loss of consciousness that are not precipitated by a specific stimulus, occur with abrupt onset and without warning, and are followed by a rapid return to baseline. A range of localizations for drop attacks is possible, but most commonly lower brainstem or spinal cord structures are implicated. Drop attacks generally indicate transient impairment of bilateral central nervous system structures involved in maintenance of postural muscle tone and balance [9]. Tumarkin otolithic catastrophes (or crises) are drop attacks without associated autonomic or neurologic symptoms in patients with severe vestibular disease, usually due to Ménière disease [10]. The causes of drop attacks include cervical cord compression, vertebrobasilar ischemia [11], inner ear disorders (e.g., Ménière disease and migrainous vertigo) [10], hydrocephalus, and myxedema [12]. We found four cases of craniospinal tumors causing drop attacks (Table 1). That includes third ventricular meningioma, fourth ventricular arachnoid cyst, and choroid plexus papilloma $[4,5,7]$. Drop attacks were attributed to the hydrocephalus caused by the lesions. One case of C2 neurinoma causing drop attacks has been reported presumably due to vertebral insufficiency [6]. Our case is fifth case of the tumor causing drop attacks and first in the region of foramen magnum. Bow Hunter's syndrome is symptomatic vertebrobasilar insufficiency due to mechanical stenosis or occlusion of a vertebral artery (VA) at C1-C2 during rotation of the head. The slow growth of tumor gives enough time for collateral pathways to develop compensating for reduced blood flow even due to dominant VA compression. Hence symptoms due to tumoral compression of vertebral arteries are rare [13]. Angiographic studies were not done to see the dominant VA in our patient due to the economic constraints. The drop attacks in the present patient could have been due to the transient vertebrobasilar insufficiency and medullary compression related to head movement as vertebral artery was encased by the tumor. Transient obstruction of flow of cerebrospinal fluid (CSF) leading to the development of an increase in intracranial pressure can also lead to drop attacks.

In elderly patients with sudden falls and presumed drop attacks, the absence of a history of loss of consciousness is unreliable. More than two-thirds of such patients are in fact found to have forms of syncope, epilepsy, and cerebrovascular disorders like vertebrobasilar insufficiency. Drop attacks due to vertebrobasilar insufficiency are commonly accompanied by other event-related neurologic manifestations (e.g., visual loss, diplopia, vertigo, and numbness) in addition to the sudden loss of postural tone in the legs [14]. Therefore ECG, EEG and relevant investigations should be done in the patients presenting with episodic falls.

Foramen magnum (FM) is bounded anteriorly by lower third of the clivus and upper edge of the body of $\mathrm{C} 2$, laterally by jugular tubercles and upper aspect of C2 laminas, posteriorly by anterior edge of the squamous occipital bone and $\mathrm{C} 2$ spinous process.

Meningiomas of Foramen magnum represent around 3\% of all meningiomas and $1 \%$ of all primary brain tumors. These represent $70 \%$ of all tumors in that region. The lesion is often large at diagnosis because of their slow-growing rate, long interval since the first symptom, and the wide subarachnoid space at this level $[1,15]$. These present with occipitocervical pain, long tract signs, and lower cranial nerve deficits $[2,3]$. Meningiomas in the foramen magnum frequently elude early diagnosis because their ill-defined symptoms mimic cervical spondylosis, multiple sclerosis, syringomyelia, normal pressure hydrocephalus, amyotrophic lateral sclerosis, Chiari I malformation, carpal tunnel syndrome, and intramedullary or extramedullary tumors [16]. Meningioma of the foramen magnum when unrecognised may lead to progressive myelopathy with quadriplegia, dysphagia, and sphincter disturbance [17].

\section{Conclusion}

Foramen magnum meningioma may rarely present with drop attacks and should be considered in the differential diagnosis of the conditions causing drop attacks. Magnetic resonance imaging (MRI) may clinch the organic causes of drop attacks and help in early diagnosis of foramen magnum meningioma. It should be included in the diagnostic workup of patients presenting with drop attacks.

\section{Conflict of Interests}

The authors declare that there is no conflict of interests regarding the publication of this paper. 


\section{References}

[1] K. I. Arnautović, O. Al-Mefty, and M. Husain, "Ventral foramen magnum meningiomas," Journal of Neurosurgery, vol. 92, no. 1, pp. 71-80, 2000.

[2] S. K. Gupta, B. S. Sharma, V. K. Khosla, S. N. Mathuriya, A. Pathak, and M. K. Tewari, "Far lateral approach for foramen magnum lesions," Neurologia Medico-Chirurgica, vol. 40, no. 1, pp. $48-52,2000$.

[3] A. Nanda, D. A. Vincent, P. S. S. V. Vannemreddy, M. K. Baskaya, and A. Chanda, "Far-lateral approach to intradural lesions of the foramen magnum without resection of the occipital condyle," Journal of Neurosurgery, vol. 96, no. 2, pp. 302-309, 2002.

[4] G. R. Criscuolo and L. Symon, "Intraventricular meningioma. A review of 10 cases of the National Hospital, Queen Square (19741985 ) with reference to the literature," Acta Neurochirurgica, vol. 83, no. 3-4, pp. 83-91, 1986.

[5] M. S. Lee, Y. C. Choi, J. H. Heo, and I. S. Choi, “Drop attacks' with stiffening of the right leg associated with posterior fossa arachnoid cyst," Movement Disorders, vol. 9, no. 3, pp. 377-378, 1994.

[6] B. George and C. Laurian, "Impairment of vertebral artery flow caused by extrinsic lesions," Neurosurgery, vol. 24, no. 2, pp. 206-214, 1989.

[7] I. F. Pollack, N. F. Schor, A. J. Martinez, and R. Towbin, "Bobblehead doll syndrome and drop attacks in a child with a cystic choroid plexus papilloma of the third ventricle. Case report," Journal of Neurosurgery, vol. 83, no. 4, pp. 729-732, 1995.

[8] M. Bringole, P. Alboni, D. Benditt et al., "Task Force on Syncope, European Society of Cardiology. Part 1. The initial evaluation of patients with syncope," Europace, vol. 3, no. 4, pp. 253-260, 2001.

[9] R. S. Maurice-Williams, "Drop attacks from cervical cord compression," British Journal of Clinical Practice, vol. 28, no. 6, pp. 215-216, 1974.

[10] A. Tumarkin, "The otolithic catastrophe: a new syndrome," British Medical Journal, vol. 2, no. 3942, pp. 175-177, 1936.

[11] M. Kameyama, "Vertigo and drop attack. With special reference to cerebrovascular disorders and atherosclerosis of the vertebral-basilar system," Geriatrics, vol. 20, no. 11, pp. 892-900, 1965.

[12] U. Kramer and A. Achiron, "Drop attacks induced by hypothyroidism," Acta Neurologica Scandinavica, vol. 88, no. 6, pp. 410411, 1993.

[13] B. George, D. Bresson, and M. Bruneau, "Extrinsic compression of the vertebral artery," in Pathology and Surgery around the Vertebral Artery, B. George, M. Bruneau, and R. F. Spetzler, Eds., chapter 20, pp. 273-283, Springer, Paris, France, 2011.

[14] J. C. M. Brust, C. R. Plank, E. B. Healton, and G. F. Sanchez, "The pathology of drop attacks: a case report," Neurology, vol. 29, no. 6, pp. 786-790, 1979.

[15] M. Bruneau and B. George, "Foramen magnum meningiomas: detailed surgical approaches and technical aspects at Lariboisière Hospital and review of the literature," Neurosurgical Review, vol. 31, no. 1, pp. 19-32, 2008.

[16] F. B. Meyer, M. J. Ebersold, and D. F. Reese, "Benign tumors of the foramen magnum," Journal of Neurosurgery, vol. 61, no. 1, pp. 136-142, 1984.

[17] W. J. Levy Jr., J. Bay, and D. Dohn, "Spinal cord meningioma," Journal of Neurosurgery, vol. 57, no. 6, pp. 804-812, 1982. 


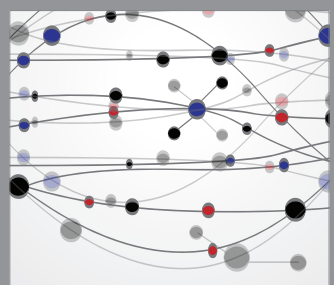

The Scientific World Journal
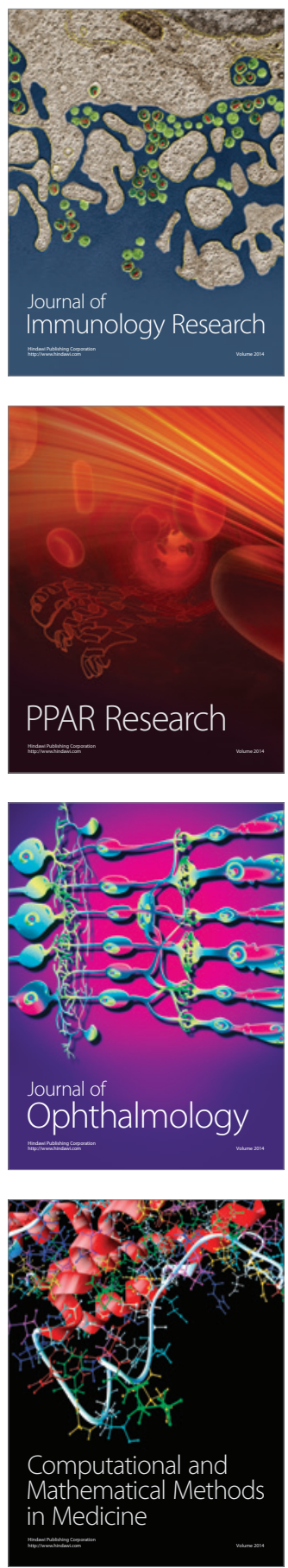

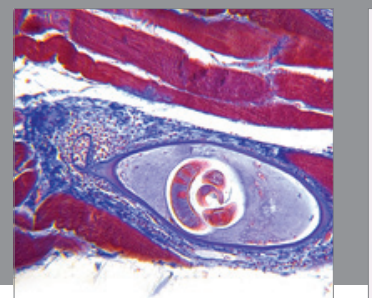

Gastroenterology

Research and Practice
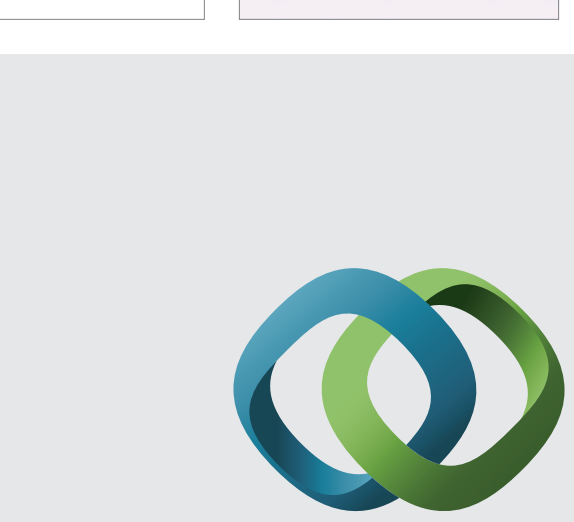

\section{Hindawi}

Submit your manuscripts at

http://www.hindawi.com
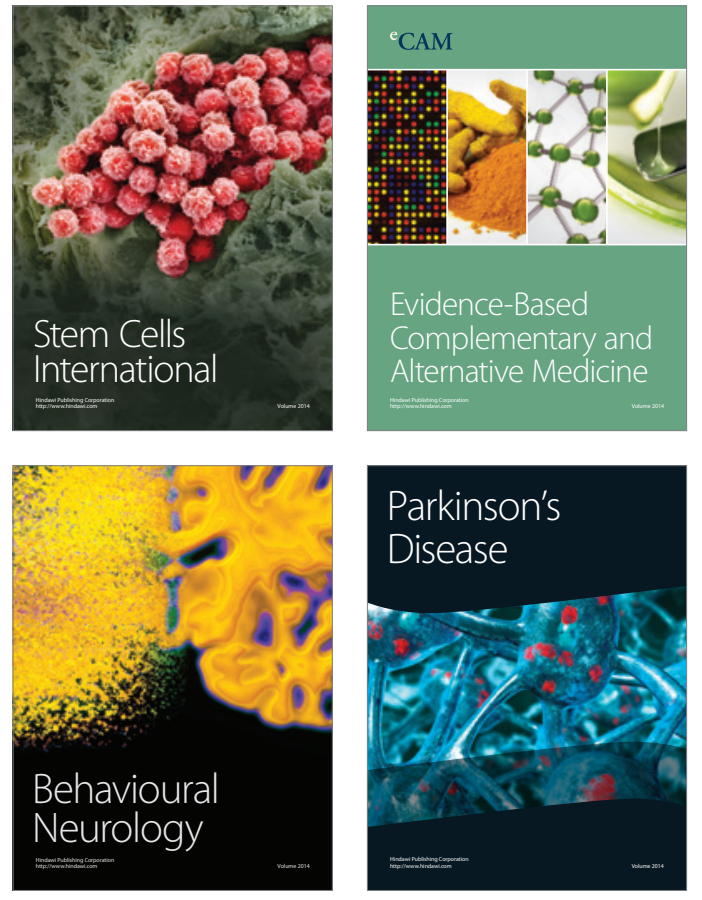
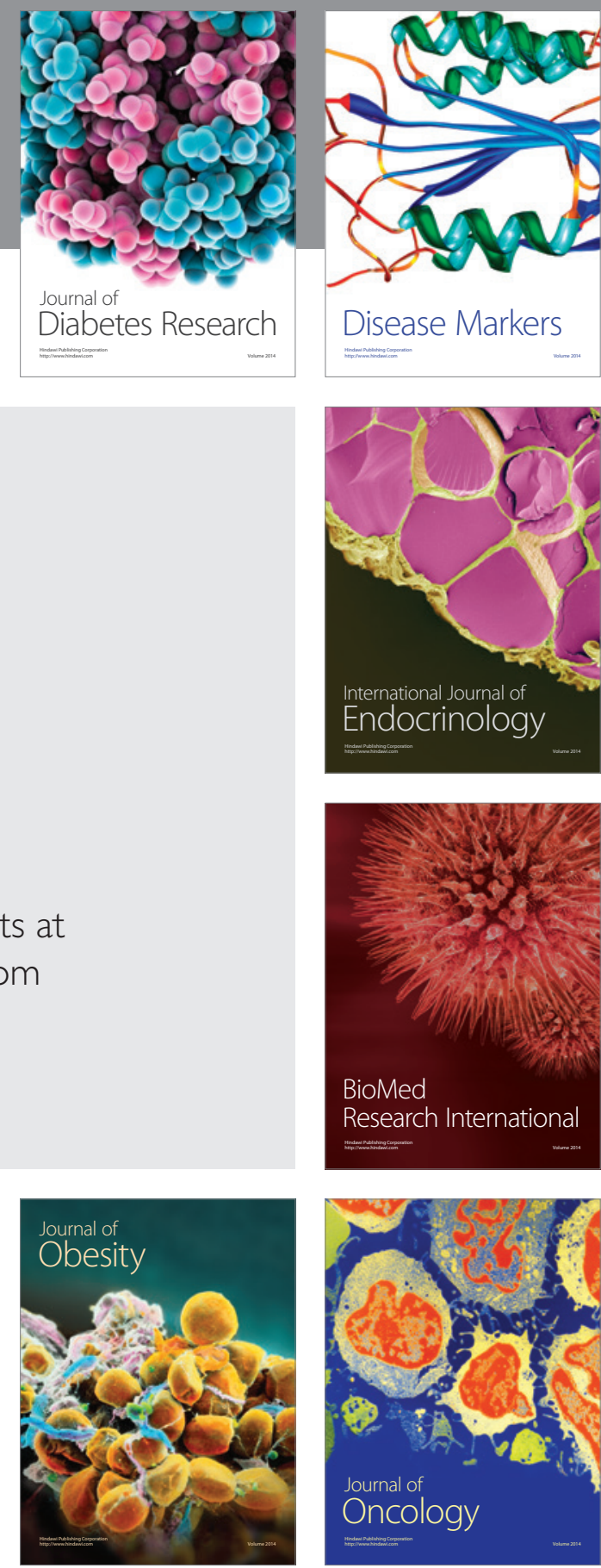

Disease Markers
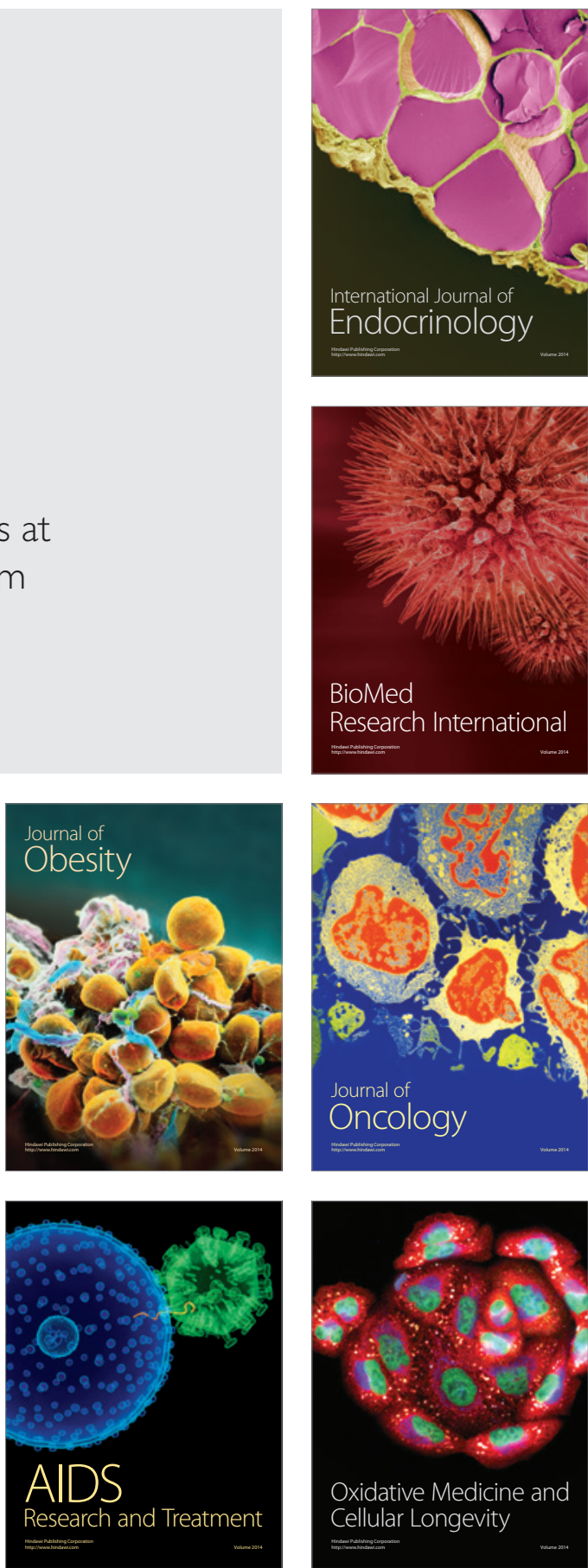